\title{
Breite Allianz fordert UMTS-Moratorium
}

\author{
Ärztinnen und Ärzte für Umweltschutz
}

Die Ärztinnen und Ärzte für Umweltschutz (AefU), unterstützt von der Verbindung der Schweizer Ärztinnen und Ärzte (FMH) und der breiten Allianz für sanften Mobilfunk*, fordern ein Moratorium für die neue Mobilfunkgeneration UMTS. Grund für das UMTS-Moratorium sind beunruhigende Ergebnisse einer aktuellen Studie aus Holland. Stellvertretend für die unterstützenden Organisationen fordern die AefU Politik, Verwaltung und Mobilfunkbetreiber auf, mit der breiten UMTS-Einführung zuzuwarten, bis die von der holländischen Studie aufgeworfenen Fragen beantwortet sind. Das Nationale Forschungsprogramm «Nichtionisierende Strahlung, Umwelt und Gesundheit» müsse nun Priorität erhalten.

\footnotetext{
* Der Allianz für sanften Mobilfunk gehören folgende Organisationen an: Ärztinnen und Ärzte für Umweltschutz (AefU), Schweizerische Energie-Stiftung (SES), Stiftung Landschaftsschutz Schweiz (SL), WWF Schweiz, Greenpeace Schweiz, Stiftung für Konsumentenschutz (SKS).
}

Korrespondenz:

Dr. med. Bernhard Aufdereggen Ärztinnen und Ärzte für Umweltschutz

Kantonsstrasse 14

CH-3930 Visp

Tel. 0279465646
Die Vorbereitungen für die Einführung des neuen Mobilfunksystems UMTS in der Schweiz laufen auf Hochtouren. Neue Antennen werden bereitgestellt, andere aufgerüstet. Denn gemäss den UMTS-Konzessionen müssen bis Ende 2004 50\% der Schweizer Bevölkerung mit UMTS-Signalen versorgt sein. Doch viel lokaler Widerstand gegen den Antennenbau führt zu Verzögerungen. Dabei wird von den Antennengegnern oft mit gesundheitlichen Problemen argumentiert. Dass diese Bedenken der Anwohnerinnen und Anwohner nicht aus der Luft gegriffen sind, zeigt eine aktuelle holländische Studie: Das renommierte TNO-Physiklabor in Holland untersuchte experimentell den Einfluss verschiedener Arten von Mobilfunksignalen auf das Wohlbefinden von Versuchspersonen. Es wurden 36 beschwerdefreie Personen untersucht sowie zusätzlich 36 Personen, die unter Elektrosmog leiden. Alle Personen wurden während 2 Stunden verschiedenen elektromagnetischen Feldern exponiert. Die Versuchspersonen wussten nicht, welchem Mobilfunksignal sie jeweils ausgesetzt waren: entweder dem Signal einer «normalen» Mobilfunkantenne (GSM), dem Signal einer UMTSAntenne oder ob der Sender abgestellt war. Die Versuchspersonen waren einer sehr geringen Strahlenbelastung ausgesetzt $(1 \mathrm{~V} / \mathrm{m})$. Im Vergleich dazu gelten in der Schweiz Anlagegrenzwerte von $6 \mathrm{~V} / \mathrm{m}$. Die holländischen Forscher erwarteten eigentlich keinen Einfluss der Strahlung auf die Versuchspersonen, sie stellten jedoch mit Erstaunen fest, dass das Wohlbefinden der Versuchspersonen signifikant abnahm, wenn sie mit einem UMTS-Signal bestrahlt wurden. Es wurde vermehrt über «Schwindel», «Nervosität», «sich nicht konzentrieren können», «Körperteile fühlen sich taub oder kribbelnd an», «leicht zerstreut sein» und weitere Beschwerden geklagt. Dies war sowohl bei den unter Elektrosmog leidenden Personen der Fall, aber auch bei gegenüber elektromagnetischen Feldern sonst beschwerdefreien Personen. Für das GSM-Signal waren die Befunde nicht eindeutig. Dass bei einer so tiefen Strahlenbelastung schon nach kurzer Zeit eine Beeinträchtigung des Wohlbefindens nachgewiesen werden konnte, ist überraschend und hat international unter Forschern Aufsehen erregt.

Bis Ende 2004 soll nun gemäss den UMTSKonzessionen 50\% der Schweizer Bevölkerung dem UMTS-Signal und damit auch den möglichen gesundheitlichen Risiken ausgesetzt werden. Die Ärztinnen und Ärzte für Umweltschutz (AefU) erachten dieses Vorgehen als nicht verantwortbar. Dies kommt einem Experiment mit $50 \%$ der Schweizer Bevölkerung gleich.

Die AefU, die FMH und die Allianz für sanften Mobilfunk fordern deshalb:

- Mit der Einführung der UMTS-Technologie in der Schweiz wird zugewartet, bis die Frage einer möglichen Beeinträchtigung des Wohlbefindens betroffener Personen geklärt ist.

- Die Eidgenössische Kommunikationskommission (ComCom) soll die UMTS-Konzessionen entsprechend abändern.

- Die Versorgungspflicht (heute 50\%) soll deutlich herabgesetzt werden. Versorgt werden sollen Wirtschaftsbereiche, die für ihre Arbeit auf diese Technologie angewiesen sind, jedoch nicht ein so grosser Teil der Bevölkerung.

- Das vorgeschlagene Nationale Forschungsprogramm «Nichtionisierende Strahlung, Umwelt und Gesundheit» ist prioritär durchzuführen, um mehr Klarheit in die gesundheitlichen Auswirkungen von Mobilfunk zu bringen. 